\title{
Human foraging behavior in a virtual environment
}

\author{
ROBERT L. GOLDSTONE and BENJAMIN C. ASHPOLE \\ Indiana University, Bloomington, Indiana
}

\begin{abstract}
We have developed an experimental platform that allows a large number of human participants to interact in real time within a common virtual world. Within this environment, human participants foraged for resources distributed in two spatially separated pools. In addition to varying the relative replenishment rate for the two pools (50-50,65-35, or 80-20), we manipulated whether the participants could see each other and the entire resource distribution or had their vision restricted to resources at their own location. Two empirical deviations from an optimal distribution of the participants were found. First, the participants were more scattered within a resource pool than the resources were themselves. Second, there was systematic underutilization of the richer pool. For example, the participants distributed themselves $73 \%$ and $27 \%$ to resource pools that had replenishment rates of $80 \%$ and $20 \%$, respectively. In addition, there were oscillations in the harvesting rate of the pools across time, revealed by a Fourier analysis with prominent power near $50 \mathrm{sec}$ per cycle. The suboptimalities and oscillations were more apparent when the locations of the participants and the food were not visible. Individual participant knowledge thus affects the efficiency with which a population of participants harvests resources.
\end{abstract}

A problem faced by all mobile organisms is how to search their environment for resources. Animals forage their environment for food, Web-users surf the Internet for desired data, and businesses mine the land for valuable minerals. When an organism forages in an environment that consists, in part, of other organisms that are also foraging, unique complexities arise. The resources available to an organism are affected not just by the foraging behavior of the organism itself, but also by the simultaneous foraging behavior of all of the other organisms. The optimal resource-foraging strategy for an organism is no longer a simple function of the distribution of resources and movement costs but is also a function of the strategies adopted by other organisms, even if the resources are replenished at a constant rate.

Our goal in the present article is to report a novel experimental technique for studying human foraging behavior. We have developed an experimental platform that allows many human participants to interact in real time within a common virtual environment. A large volume of time-varying data can be collected from participants as they vie for resources. Two resource pools were created within this environment, and we recorded the momentby-moment exploitation of these resources by each human participant.

This research was funded by NIH Grant MH56871 and NSF Grant 0125287. A preliminary report of the results from the experiment were presented at the 25th Annual Conference of the Cognitive Science Society. The authors thank Jerry Busemeyer, Jason Gold, Michael Roberts, Nathan Steele, William Timberlake, Peter Todd, and Michael Wade for helpful suggestions on this work. Correspondence concerning this article should be addressed to R. L. Goldstone, Psychology Department, Indiana University, Bloomington, IN 47405 (e-mail: rgoldsto@indiana.edu).
We used this environment to address research questions concerning the allocation of foragers to resource pools. We explored how resource-foraging strategies unfold with time, the nature of systematic deviations from optimality in resource foraging, and how participants' information about the distribution of resources and other participants affects their aggregate distribution. In particular, previous research has shown that the distribution of foragers to resources is often not as extreme as the distribution of the resources themselves (Kennedy \& Gray, 1993; Sokolowski, Tonneau, \& Freixa i Baqué, 1999). We tested whether this was true for our virtual environment and whether giving agents moment-by-moment information about the forager and resource distributions would promote more effective foraging (Templeton \& Giraldeau, 1996). In addition, we assessed whether periodic cycles underlaid migrations into and out of resource pools. Although there is a long history of studying population cycles in the dynamics of predator-prey systems (Lotka, 1925), much less is known about the conditions under which a single population harvesting resources from constantly replenishing patches will show cyclic population changes.

\section{Foraging in Groups}

One model in biology for studying the foraging behavior of populations is ideal free distribution (IFD; Fretwell \& Lucas, 1970; Seth, 2001). This model assumes that animals distribute themselves among patches so as to maximize the gained resources. The specific assumptions of the model are that animals (1) are free to move between resource patches without cost, (2) have correct ("ideal") knowledge of the rate of food occurrence at each patch, and (3) are equal in their abilities to 
compete for resources. The model predicts an equilibrium distribution of foragers such that no forager can profit by moving elsewhere. This condition is met if the distribution of foragers matches the distribution of resources across patches. For example, if the resource amounts in two patches are $N_{\mathrm{A}}$ and $N_{\mathrm{B}}$ and the numbers of foragers occupying the two patches are $F_{\mathrm{A}}$ and $F_{\mathrm{B}}$, the IFD model predicts the ratio of $N_{\mathrm{A}}$ to $N_{\mathrm{B}}$ to be equal to the ratio of $F_{\mathrm{A}}$ to $F_{\mathrm{B}}$. In practice, conformity of the data to the IFD model can be evaluated through the following equation:

$$
\log \left(\frac{F_{\mathrm{A}}}{F_{\mathrm{B}}}\right)=s \log \left(\frac{N_{\mathrm{A}}}{N_{\mathrm{B}}}\right)+b,
$$

where $s$ is a parameter that controls the sensitivity of foragers to variations in the resource distribution and $b$ is a bias parameter (see Kennedy \& Gray, 1993; cf. Baum, 1974). The IFD model predicts the best-fitting values of $s$ and $b$ to be 1 and 0 , respectively.

Consistent with this model, groups of animals often distribute themselves in a nearly optimal manner, with their distribution matching the distribution of resources. For example, Godin and Keenleyside (1984) distributed edible larvae to two ends of a tank filled with cichlid fish. The food was distributed in ratios of 1:1, 2:1, or 5:1. The cichlids quickly distributed themselves in rough accord with the relative rates of the food distribution before many of the fish had even acquired a single larva and before most of the fish had acquired larvae from both ends. Similarly, Harper (1982) observed that mallard ducks distributed themselves in accord with the rate or amount of food thrown at two pond locations. When one patch produced two times the amount of resource as another patch, there were approximately two times as many ducks at the richer patch, relative to the leaner one.

Although animals frequently distribute themselves in approximate accord with an IFD, systematic deviations have also been observed. One common result is undermatching, defined as a distribution of animals that is less extreme than the distribution of resources (Kennedy \& Gray, 1993). Undermatching is obtained in Equation 1 when $s$ is less than 1; an example would be a $75 \%: 25 \%$ distribution of foragers when the resources have a 80\%:20\% distribution. When undermatching occurs, there are fewer animals at the richer patch and more animals at the leaner patch than is optimal. Overmatching is obtained when $s$ is greater than 1 and entails a distribution of animals that is more extreme than the distribution of resources. The few experiments in which group foraging behavior with humans has been examined have also shown undermatching (Kraft \& Baum, 2001; Madden, Peden, \& Yamaguchi, 2002; Sokolowski et al., 1999). In some of these experiments (Kraft \& Baum, 2001; Madden et al., 2002), each participant in a group held up a card indicating one of two colors, and then one card was probabilistically selected as the rewarded card. The participants who held up the rewarded color shared the rewards. In another paradigm (Madden et al., 2002), participants moved between two designated areas and shared rewards that were probabilistically deposited in these areas. In both paradigms, greater average amounts of reward were obtained by the participants at the richer patch, indicating undermatching. Several accounts for human and animal undermatching have been suggested. One possibility is that undermatching is caused by increased foraging interference for high-density crowds (Kennedy \& Gray, 1993). Another possibility is that organisms may differ in their competitive abilities, with competitively fitter organisms "hogging" resources at the richer patch (Houston \& McNamara, 1988). A third explanation is that organisms may distribute themselves so that their numbers match the difference, rather than the ratio, of resource outputs (Sokolowski et al., 1999).

Our experiment extends the previous studies of group foraging in humans in a few directions. First, we have developed a computer-based platform for the foraging experiment that allows us to manipulate experimental variables that would be difficult to manipulate in a more naturalistic environment. Second, we collect second-bysecond data on the amount of resources and number of participants at different pools, which allows us to explore variation in resource use with high temporal resolution. Third, although our environment is virtual, it is naturalistic in one important respect: Resources are distributed in a continuous spatial environment, rather than at two discrete locations. Fourth, we do not designate or identify the resource alternatives to participants. As in many natural situations (Kennedy \& Gray, 1993), the participants must discover the number and locations of resource patches themselves.

\section{Resource Distribution and Visibility}

Using our virtual environment with interacting participants, we manipulated the relative outputs of the different resource pools and the knowledge possessed by the agents. Manipulating the relative outputs of pools allows stable estimates of undermatching to be obtained. If a constant degree of undermatching occurs, a good fit of Equation 1 to the empirical data should be found by estimating the slope $s$ and the intercept $b$, using least mean square deviation (Kennedy \& Gray, 1993).

The second experimental variable that we manipulated was the participants' knowledge of their environment and other participants. In Godin and Keenleyside's (1984) experiment with cichlids, every cichlid could see the other cichlids as well as the larvae resources at both ends of the tank. Gallistel (1990) argued that this kind of information is important for the cichlids to distribute themselves rapidly in accord with the resource distribution. They learn about the resource distributions by observing events that do not directly involve themselves. However, in individual reinforcement learning situations, an agent has access only to the outcomes of its own actions. It does not have access to the values of options not selected. Both situations occur naturally, and it is possible that the ability of a group to efficiently distribute itself to resources 
depends on the information at each agent's disposal (Templeton \& Giraldeau, 1996).

\section{EXPERIMENT}

We have developed a software system that records the instant-by-instant actions of individuals foraging for resources in a shared environment. Two resource pools were created with different rates of replenishment. The participants' task was to obtain as many resource tokens as possible during an experiment. A participant obtained a token by being the first to move on top of it. In addition to varying the relative replenishment rates for the two pools $(50-50,65-35$, or $80-20)$, we manipulated whether the participants could see each other and the entire food distribution, or had their vision restricted to food at their own location.

\section{Method}

One hundred sixty-six undergraduate students from Indiana University served as participants in order to fulfill a course requirement. The students were run in eight groups with 21, 20, 23, 19, 28, 12,25 , and 18 participants. Each student within a group was assigned to a PC computer in a large computer-based classroom with 40 computers. The experimenter controlled the experiment from another computer in the same room. The participants' computers were registered with the experimenter's computer, using our developed software for sending messages over the Internet to a set of networked computers.

The participants were asked to participate in an experiment on group behavior. They were instructed to try to pick up as many "food" pieces as possible by moving their icons' position on top of food locations. The participants were told that the food would occur in clumps and that, if they learned where the productive clumps were, they could harvest more food. Explicitly telling the participants that there were clusters of food deviates from many natural foraging situations in which animals must inductively learn that food is clustered but was judged expedient in the present experiment, given its brevity.

The participants were also told that there would be a lottery at the end of the experiment to win $\$ 10$ and that every piece of food they collected during the experiment would be worth one lottery ticket. In this manner, the participants were motivated to collect as many pieces of food as possible, even if at some point in the experiment they felt that it was improbable that they could collect more food than any other participant. The participants were told to look only at their own computer screen and not to talk with each other at any time.

The participants within a group coexisted in a virtual environment made up of replenishing resource pools and other participants. The environment consisted of an $80 \times 80$ grid of squares. The participants controlled their position within this world by moving up, down, left, and right, using the four arrow keys on their computers' keyboards. A participant could not walk off one side of the grid and reappear on the other. Each participant was represented by a yellow dot. Food was gathered when a participant's position coincided with a piece of food. In the visible condition, all of the other participants' locations were represented by blue dots, and available food resources were represented by green dots. Thus, the participants had access to the moment-by-moment locations of every food piece and participant. When food was harvested by a participant, it immediately disappeared from the screen. In the invisible condition, each participant saw only his or her own position on the screen (as represented by a yellow dot) and any food gathered in the last $2 \mathrm{sec}$ (as represented by a green dot). After this time interval, these con- sumed food pieces disappeared. Thus, the participants in the invisible condition saw only their own location and, occasionally, would see green dots appear when they successfully harvested pieces of food.

Every experiment was divided into six 4.5 -min sessions. These six games consisted of all combinations of the two levels of knowledge (visible vs. invisible) and the three levels of resource distribution $(50 / 50,65 / 35$, or $80 / 20)$. For each of the three distribution conditions, two resource pools were constructed, with center locations at reflections and rotations of the set of coordinates $\{40,15\}$ and $\{15,65\}$. A different reflection and rotation was used for each of the six conditions, with the result that the resource centers were approximately equally likely to be in each of eight possible locations and the two centers within one session always had the same distance from one another. Two opposite orders of the six games were randomly assigned to the eight separate groups of participants.

A counter in the lower left-hand portion of each participant's screen indicated the cumulative number of food pieces collected by the participant in the session. The rate of distribution of food was based on the number of participants, with one piece of food delivered every $4 / N \mathrm{sec}$, where $N$ is the number of participants. When a piece of food was delivered, it was assigned to a pool probabilistically on the basis of the distribution rate. For example, for the 80/20 condition, the food would occur in the richer pool $80 \%$ of the time and in the leaner pool $20 \%$ of the time. The location of the food within the pool followed a Gaussian distribution with a mean at the center of the pool and a standard deviation of five horizontal and vertical positions. Thus, the probability of food occurring in a given location was inversely related to the distance between the location and the pool's center. The one exception to this was that no piece of food was placed on top of another piece of food. If the randomly selected location for a new piece of food coincided with an existing piece of food, a new (Gaussian) random location for the food was selected within the same pool. Food could be placed on top of a participant, in which case the food counter would indicate to the participant that he or she had received a food piece. Multiple participants could occupy the same location without colliding. Any food that was simultaneously found by 2 or more participants was randomly assigned to one of them. All the food remained on the screen until it was consumed by a participant.

Data were recorded every $2 \mathrm{sec}$ that included the positions of all the participants, the number of food pieces collected by each participant, and the locations of uncollected food pieces. After all six sessions were completed, a winning participant was selected to receive $\$ 10$ by an automatic lottery. The probability of a participant's winning the lottery was equal to the number of food pieces he or she collected, divided by the total number of food pieces collected by all the participants.

\section{Results}

As a preliminary analysis of the distribution of participants across resource pools, Figure 1 shows the frequency with which each of the $80 \times 80$ grid cells was visited by participants in each of the six experimental conditions. The brightness of a cell increases proportionally with the number of times the cell was visited. The few isolated white specks can be attributed to participants who decided not to move for extended periods of time. In Figure 1, the thick and thin circles show two standard deviations of the food distribution for the richer and the leaner pools, respectively. An inspection of this figure indicates that the participants spent the majority of their time within relatively small regions centered on the two resource pools. For the invisible conditions, there was substantial diffusion of travel outside of one stan- 

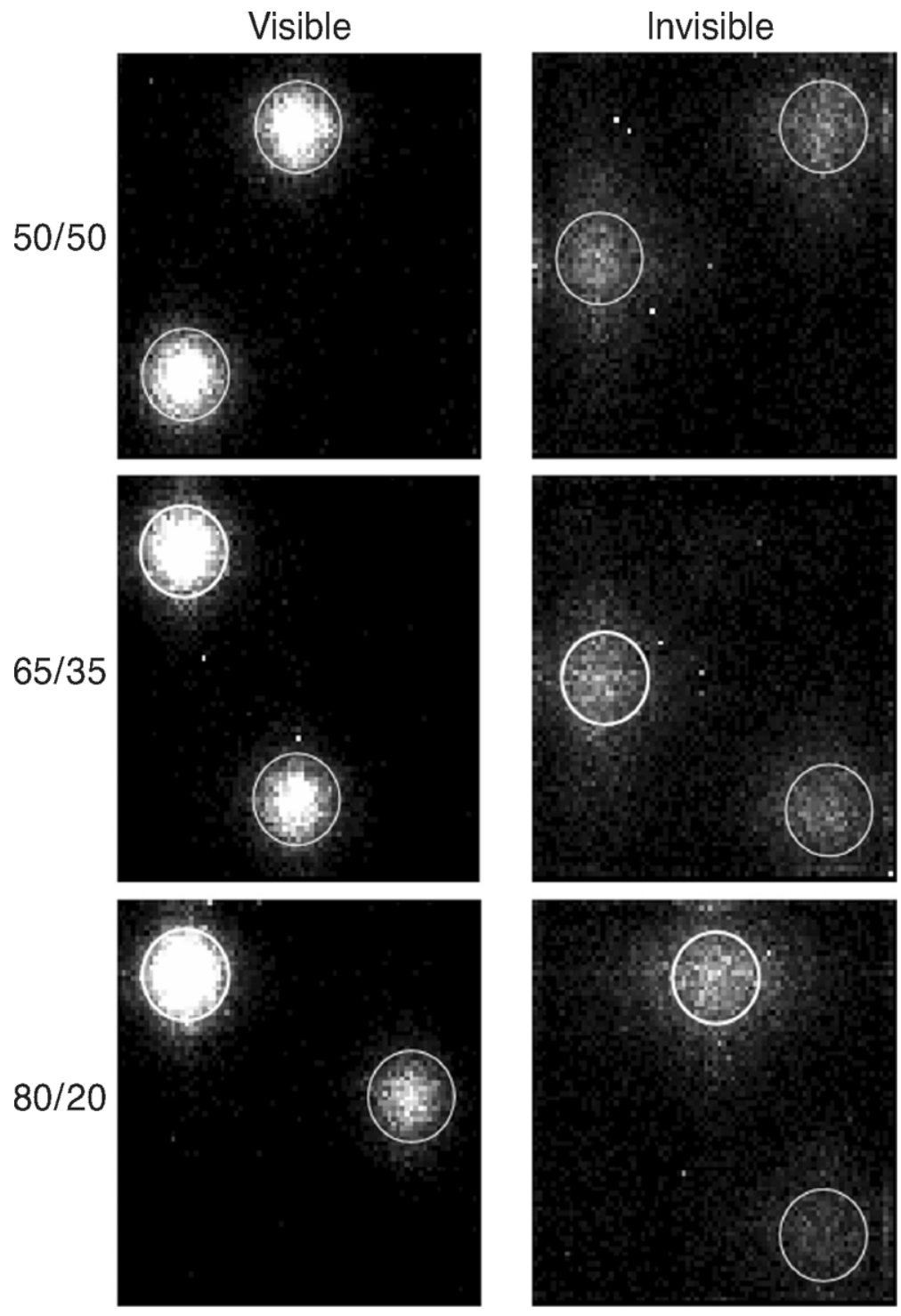

Figure 1. A frequency plot of participants' visits to each grid square.

dard deviation of the pools' centers. A Cochran's test for homogeneity of variances revealed significantly greater variability for the invisible than for the visible condition $(p=.0004)$, indicating greater scatter of the participants' locations in the invisible condition. The influence of increased time within a session on the reduction of variability was significant in the invisible (Pearson's $r=$ $-.24, p=.003)$, but not the visible $(r=-.03, p=.41)$, condition. The participants approximately distributed themselves in a Gaussian form.

The dynamics of the distribution of participants to resources is shown in Figure 2, broken down by the six conditions. In this figure, the proportion of participants in the two pools is plotted over time within a session. Horizontal lines indicate the proportions that would match the distribution of food. A participant was counted as residing in a pool if he/she was within five food distribution standard deviations of the pool's center. This created circular pools that were as large as possible without overlapping. ${ }^{1}$ The participants who were not in either pool were excluded from Figure 2, and the total number of participants was normalized to exclude these participants. Although Figure 2 shows that the distribution of participants adjusted quickly, including the earliest time samples in the probability distribution estimate would lead to estimates that were inappropriately regressed toward the mean of $50 \%$. For this reason, our estimates were obtained from the 50- to 270 -sec range (we also analyzed results from the 150 - to 270 -sec range, and these results did not differ significantly from the 50- to 270sec range). The distribution of participants systematically undermatched the optimal probabilities. For the $65 / 35$ distribution, the $65 \%$ pool attracted an average of $60.6 \%$ of the participants in the 50 - to 270 -sec interval 


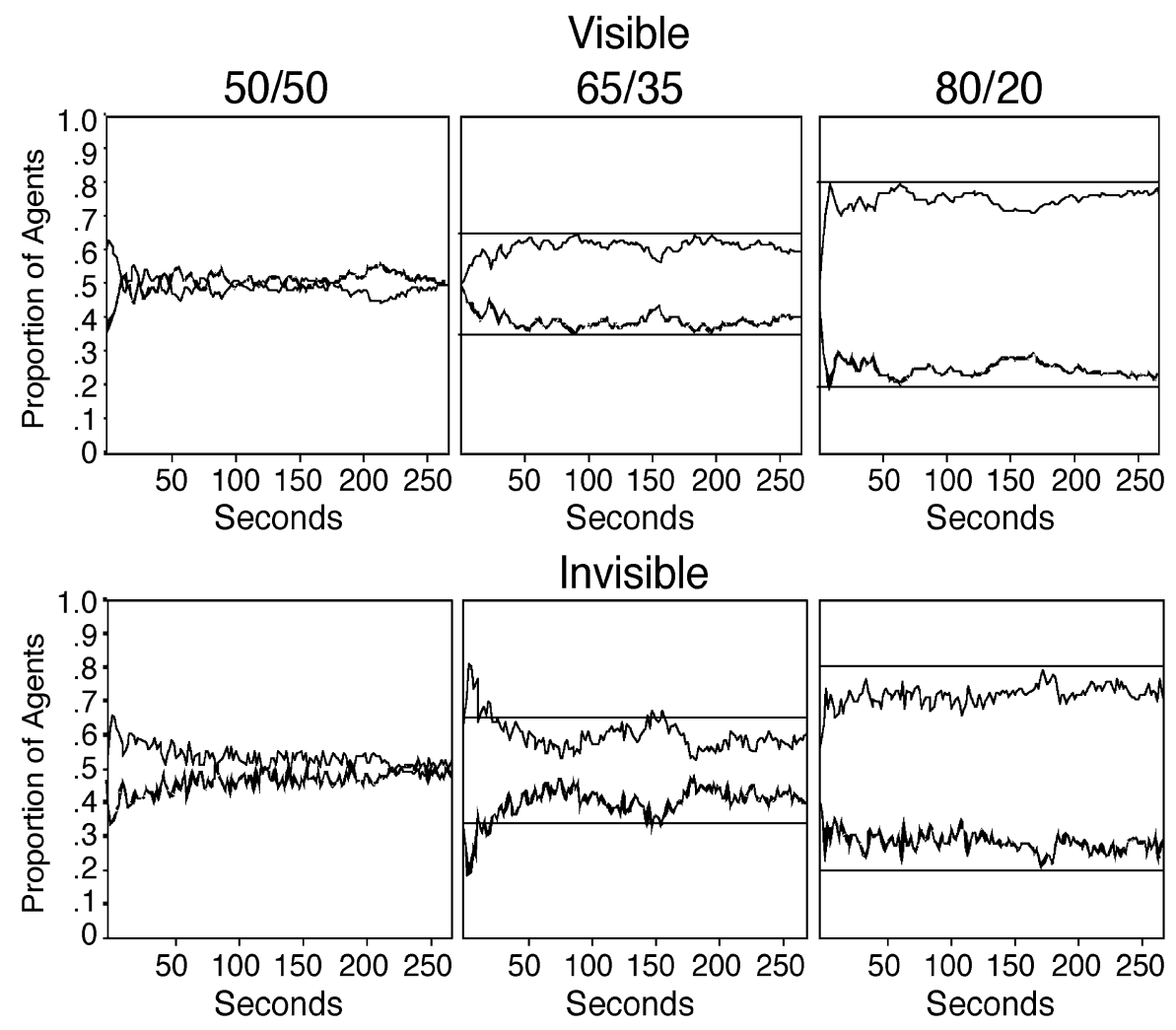

Figure 2. Changes in group sizes over the course of a session.

across both the visible and the invisible conditions. The likelihood that the true population percentage was less than $65 \%$ is $99.6 \%$, according to a Bayesian analysis with flat priors $[t(7)=3.9$; Berry, 1996]. Similarly, for the $80 / 20$ distribution, the $80 \%$ pool attracted only $73.5 \%$ of the participants, and given this data, the probability that the true population percentage in the $80 \%$ pool was less than $80 \%$ is $99.6 \%[t(7)=4.3]$.

An effective way to measure the overall amount of undermatching or overmatching of foragers to resources across a set of resource distributions is to find the bestfitting values of $s$ and $b$ in Equation 1 to the distributions. Figure 3 shows the relation between the logarithm of the ratio of resource distributions and the logarithm of the ratio of participant distributions. If a consistent degree of undermatching is found across resource distributions, a linear relation with a slope less than 1 should provide a good fit. Using a least mean square deviation method, the best-fitting values for $s$ and $b$ are 0.78 and 0.01 , respectively, in the visible condition and 0.68 and 0.01 , respectively, in the invisible condition. Both of these linear relations account for at least $98 \%$ of the variance in the participant distributions. Across the eight groups, the best-fitting $s$ values ranged from 0.57 to 0.94 for the visible condition and from 0.55 to 0.81 for the invisible condition. The values for $s$ are below 1, indicating undermatching in both visibility conditions, and are close to the average value of $s=0.7$ given in Kennedy and Gray's (1993) meta-analysis of 52 studies. A Bayesian analysis indicates a 95\% likelihood that the true population $s$ value for the invisible condition is greater than the $s$ value for visible condition $[t(7)=2.4]$. The percentage of participants falling outside of either pool during the $50-270 \mathrm{sec}$ interval are $1.2 \%$ and $13.4 \%$ for the visible and the invisible conditions, respectively.

A final analysis of interest explores the possibility of periodic fluctuations in resource use. Informal observations suggested the occurrence of cycles of overuse and underuse of pools. The participants seemed to congregate heavily at a pool for a period of time and then migrate from this pool to the other pool. If a fairly large subpopulation within a pool decides at roughly the same time to migrate from one pool to another, cyclic waves of population change may emerge. This was tested by applying a Fourier transformation to the data shown in Figure 2. Fourier transformations translate a time-varying signal into a set of sinusoidal components. Each sinusoidal component is characterized by a phase (where it crosses the $y$-intercept) and a frequency. In a frequency plot, the power at a frequency indicates the strength of a periodic response at that frequency.

Any periodic waves of population change that occur in the experiment would be masked in Figure 2 because the graphs average over eight different groups of participants. Accordingly, four steps were taken to produce the frequency plot shown in Figure 4. First, we derived a data vector of the proportion of participants in the richer pool across a 4.5-min session for each of the eight groups within 


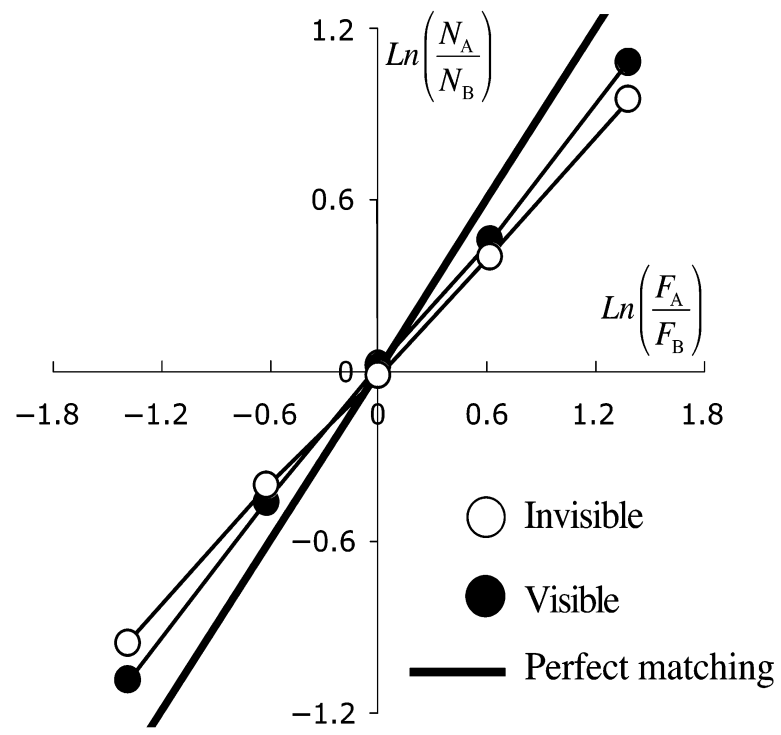

Figure 3. Comparison of resource distributions at Patches $A$ and $\mathrm{B}\left(N_{\mathrm{A}}\right.$ and $\left.N_{\mathrm{B}}\right)$ to forager distributions at these patches $\left(F_{\mathrm{A}}\right.$ and $\left.F_{\mathrm{B}}\right)$.

each of the six conditions. Second, we detrended each vector by removing the best straight-line fit from it. Third, we applied a digital Fourier transformation (fast-Fourier transform) to each detrended vector. Fourth, we created the frequency plots in Figure 4 by averaging the frequency plots for the eight groups within a single condition.

The resulting frequency plots show significantly greater power in the low-frequency spectra for invisible than for visible conditions. In particular, the average power for frequencies up to 0.05 cycles is 3.4 and 1.1 for the invisible and the visible conditions, respectively. Treating each session's average spectral power as a single data point, the likelihood of the true power's being greater in the invisible than in the visible condition is $99.8 \%$, assuming flat priors $[t(7)=4.1]$. The power in lower frequencies is particularly high for the invisible condition with an $80 / 20$ distribution. For all three invisible conditions, the peak power is at approximately 0.02 cycles/sec. This means that the participants tend to have waves of relative dense crowding at one pool that repeat about once every $50 \mathrm{sec}$. This 50 -sec period includes the time both to migrate from the first pool to the second pool and to return to the first pool. A pronounced power peak at lower frequencies is absent for the visible condition. In the visible condition, each participant can see whether the other participants are closer than themselves to underexploited resource pools. This information apparently affects the likelihood of the participants' shifting between pools. Participants may be less likely to shift from Pool A to Pool B if they see that other participants have already departed for Pool B. In the invisible condition, the lack of information about the locations of other participants means that an agent would not see that other agents have already left their pool. Consistent with this account, the overall rate of pool switching is greater in the invisible than in the visible condition (average switch rates of 8.5 vs. 3.3 times per session, respectively). In addition, there was greater variability of net in-flow and out-flow of the participants in the invisible than in the visible condition. The net change in the richer pool's population was calculated for a moving 10 -sec window across each 4.5 -min experiment. This net change had an average variance of 5.5 versus 1.2 for the invisible and the visible conditions, respectively. Monte Carlo simulations indicated that for the invisible, but not the visible, condition, the population variability was greater than would be predicted if each participant had switched pools independently of the other participants. In other words, the participants' movements in and out of pools were partially synchronized in the invisible condition, yielding relatively large population fluctuations.

It is less clear why periodic population waves should be greatest for the most lopsided, $80 / 20$ distribution, but one speculation is that the slowly replenishing $20 \%$ pool has the least power to attract a stable population of participants. A few participants can come into the $20 \%$ pool and quickly exhaust all of the fallen food resources. Then the slow replenishment rate gives all of the participants in the pool little incentive to stay, and they consequently move to the $80 \%$ pool, until eventually the $20 \%$ pool becomes attractive again because of its low population density and cache of accumulated food.

\section{Discussion}

The results of the present experiment indicate systematic inefficiencies in the distribution of human participants to resources over time. First, participants exhibited undermatching, in the sense that there were too many participants at the leaner resource pool and too few participants at the richer pool. If this result proves to be general, participants in similar situations ought to increase their use of relatively rich pools despite the possibly greater population density at those pools. The task constraints and evidence for undermatching in both visible and invisible conditions argue against undermatching being due to either resource interference or inaccurate perception of resource quantities. Our ongoing modeling work suggests that people have a tendency to sample all discovered resource pools with equal frequency and that this tendency combines with a tendency to sample pools with greater reward outputs. Sampling pools approximately evenly is a useful strategy in an environment in which resource outputs may vary with time (Bell \& Baum, 2002).

Second, systematic cycles of population change are apparent whereby the migration of people from one pool to another is roughly synchronized. Synchronized population waves are disadvantageous for those participants moving in the wave, because competitive crowding results in decreased food intake. The present study is the first to report this cyclic foraging behavior in humans. However, Madden et al. (2002) have reported resourceswitching behavior that may have been caused by similar individual decisions. They found that participants who received relatively low levels of reward on a given 


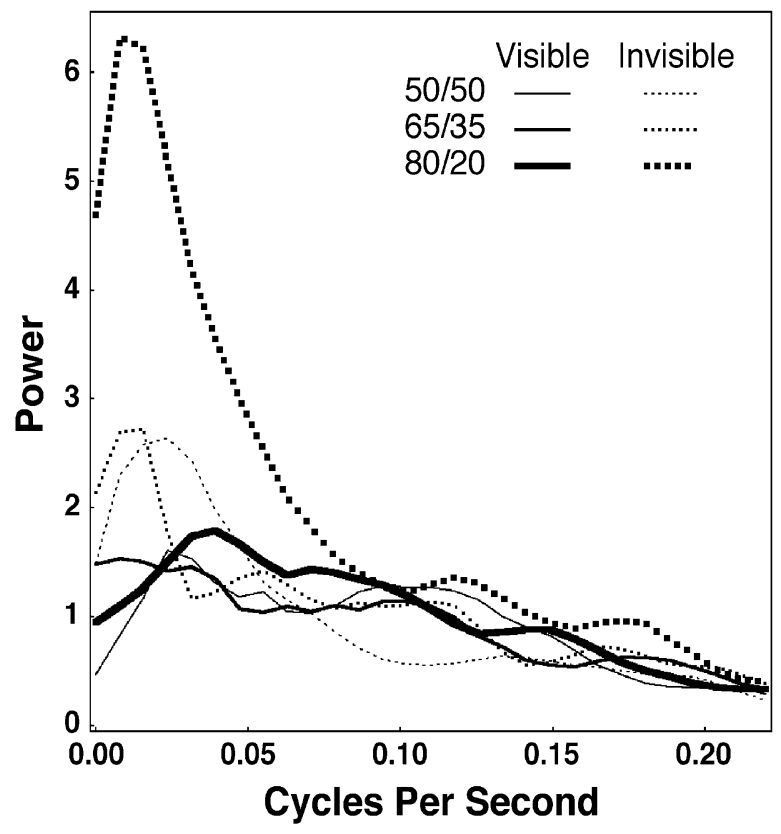

Figure 4. A Fourier analysis of group size over time.

trial were relatively likely to switch pools on the subsequent trial. This lose-shift strategy may also have been the cause for our population waves.

The third result is that the participants were more scattered than were the food resources. Both the participants and the food were distributed in a roughly Gaussian form, but the positional variance associated with the participants was higher. The participants often looked for resources in areas that were never reinforced themselves but were close to reinforced areas.

All three of these effects were more pronounced for invisible than for visible conditions. In fact, of the three effects described above, the only one that was appreciably large in the visible condition was the systematic undermatching. The influence of visibility suggests that an individual's knowledge of the moment-by-moment state of the environment and other participants can allow the group as a whole to avoid inefficient waves of resource under- and overexploitation.

A fruitful extension to the present work would be the development of an agent-based computer simulation that models the large quantity of spatial-temporal population information amassed in the experiment (Seth, 2001). Such a model would need to incorporate a distinction between agents with and without vision. Blind agents might resemble standard reinforcement learning devices if supplemented by processes that undermatch distributions. Incorporating agents with vision is more challenging, raising important issues with regard to generating expectations and planning. However, the present empirical work suggests that developing these more sophisticated agents with knowledge is worth the trouble. Knowledge of food distributions allows an agent to more effectively match those distributions, whereas knowledge of other agents allows an agent to more effectively decouple its responses from others.

\section{REFERENCES}

BAUM, W. M. (1974). On two types of deviation from the matching law: Bias and undermatching. Journal of the Experimental Analysis of Behavior, 22, 231-242.

BELL, K. E., \& BAUM, W. M. (2002). Group foraging sensitivity to predictable and unpredictable changes in food distribution: Past experience or present circumstances? Journal of the Experimental Analysis of Behavior, 78, 179-194.

BERRY, D. A. (1996). Statistics: A Bayesian perspective. Belmont, CA: Wadsworth.

Fretwell, S. D., \& LuCAs, H. L. (1970). On territorial behavior and other factors influencing habitat distribution in birds. Acta Biotheoretica, 19, 16-36.

GALLISTEL, C. R. (1990). The organization of learning. Cambridge, MA: MIT Press, Bradford Books.

Godin, J.-G. J., \& KeENLEYside, M. H. A. (1984). Foraging on patchily distributed prey by a cichlid fish (Teleostei, Cichlideae): A test of the ideal free distribution theory. Animal Behaviour, 32, 120-131.

HARPER, D. G. C. (1982). Competitive foraging in mallards: "Ideal free" ducks. Animal Behaviour, 30, 575-584.

Houston, A. I., \& McNamara, J. M. (1988). The ideal free distribution when competitive abilities differ: An approach based on statistical mechanics. Animal Behaviour, 36, 166-174.

Kennedy, M., \& Gray, R. D. (1993). Can ecological theory predict the distribution of foraging animals? A critical analysis of experiments on the ideal free distribution. Oikos, 68, 158-166.

KrafT, J. R., \& BAUM, W. M. (2001). Group choice: The ideal free distribution of human social behavior. Journal of the Experimental Analysis of Behavior, 76, 21-42.

LOTKA, A. J. (1925). Elements of physical biology. Baltimore: Williams \& Wilkins.

Madden, G. J., Peden, B. F., \& Yamaguchi, T. (2002). Human group choice: Discrete-trial and free-operant tests of idea free distribution. Journal of the Experimental Analysis of Behavior, 78, 1-15.

$\mathrm{SETH}$, A. K. (2001). Modeling group foraging: Individual suboptimality, interference, and a kind of matching. Adaptive Behavior, 9, 67-91.

Sokolowski, M. B. C., Tonneau, F., \& Freixa i Baqué, E. (1999). The ideal free distribution in humans: An experimental test. Psychonomic Bulletin \& Review, 6, 157-161.

TEMPLETON, J. J., \& GiRALDEAU, L. A. (1996). Vicarious sampling: The use of personal and public information by starlings foraging in a simple patchy environment. Behavioral Ecology \& Sociobiology, $\underline{38}_{2}$ 105-114.

\section{NOTE}

1. Analyses were also conducted using two, rather than five, standard deviations as a cutoff. This naturally reduced the numbers of participants counted as occupying the pools but did not otherwise affect the results reported here.

(Manuscript received October 22, 2002; revision accepted for publication May 12, 2003.) 